\title{
Perinatal outcome in uncomplicated pregnancies with oligohydramnios
}

\author{
Snehal Gaware*, V. B. Bangal
}

Department of Obstetrics and Gynecology, Pravara Rural Medical College, Ahmednagar, Maharashtra, India

Received: 22 October 2018

Accepted: 28 November 2018

\author{
*Correspondence: \\ Dr. Snehal Gaware, \\ E-mail: snehalv91@gmail.com
}

Copyright: ( $\odot$ the author(s), publisher and licensee Medip Academy. This is an open-access article distributed under the terms of the Creative Commons Attribution Non-Commercial License, which permits unrestricted non-commercial use, distribution, and reproduction in any medium, provided the original work is properly cited.

\begin{abstract}
Background: Oligohydramnios refers to amniotic fluid volume that is less than expected for gestational age. We aimed to assess the perinatal outcomes in pregnancies with oligohydramnios.

Methods: The prospective study was conducted in the Department of Obstetrics and Gynecology, Pravara Medical College, Loni in which 200 consecutive singleton pregnancies delivered vaginally, with intact membranes were included. Amniotic fluid index was determined using the Phelan's technique at the time of admission and women were diagnosed with oligohydramnios if AFI was five or less, which formed the first group and the rest of mother formed the second group. Perinatal outcomes were noted in the proforma as well.

Results: Of the 200 mothers included in the study, 38 had AFI $\leq 5$. Baseline characteristics was similar in both the groups. Most common antenatal risk factors studies were pregnancy induced hypertension (29\% vs 12\%; p value $<0.05)$, intrauterine growth restriction ( $34 \%$ vs $10 \%$; $p$ value $<0.001)$ and severe anemia $(21 \%$ vs $9 \%$; $p$ value <0.05). Proportion of pregnancies needing induction of labor and birth weight less than $2.5 \mathrm{kgs}$ were significantly higher among mothers with oligohydramnios.

Conclusions: Authors observed that induction of labor and low birth weight were significantly associated with oligohydramnios. Prospective randomized trials are needed to establish whether early induction of labor in the presence of a oligohydramnios improves perinatal outcome.
\end{abstract}

Keywords: Amniotic fluid index, Fetal outcome, Oligohydramnios

\section{INTRODUCTION}

Oligohydramnios refers to amniotic fluid volume that is less than expected for gestational age. The volume of amniotic fluid is ultimately determined by the volume of fluid flowing into and out of the amniotic sac.

Fetal urination, lung fluid, and swallowing all make important contributions to fluid movement in late gestation, with minimal contributions from other sources. Fetal disorders that affect any of these processes will affect the amniotic fluid volume. It is recommended that amniotic fluid volume should be assessed either qualitatively or quantitatively at every antenatal ultrasound examination. Although use of an objective criterion is generally preferable, subjective suspicion of amniotic fluid volume may also be done. The amniotic fluid index (AFI) measurement is calculated by first dividing the uterus into four quadrants using the linea nigra for the right and left divisions and the umbilicus for the upper and lower quadrants. The maximum vertical amniotic fluid pocket diameter in each quadrant not containing cord or fetal extremities is measured in centimeters; the sum of these measurements is the AFI. According to the common convention, AFI less than $5 \mathrm{~cm}$ is considered as oligohydramnios, and has been associated with a variety of adverse perinatal outcomes such as perinatal death, nonreassuring fetal heart rate 
during labor, meconiam-stained fluid and admission of the neonate to the intensive care unit. ${ }^{1}$ However, recent literature suggests that isolated oligohydramnios is not related with worse perinatal outcomes than pregnancies with normal amniotic fluid. ${ }^{2} \mathrm{We}$ aimed to assess the perinatal outcomes in pregnancies with oligohydramnios.

\section{METHODS}

\section{Study design and sampling}

The prospective study was conducted in the Department of Obstetrics and Gynecology, Pravara Medical College, Loni in which 200 participants were enrolled from January 2018 till July 2018. We included consecutive singleton pregnancies delivered vaginally, with intact membranes. Women with premature rupture of membranes, known fetal anomalies, gestational diabetes, placental anomalies, any medical illness and multiple pregnancies were excluded from the study. Patients were explained the purpose of the study and an informed written consent was obtained from them. The study was approved by the institutional ethics committee. Amniotic fluid index was determined using the Phelan's technique at the time of admission and women were diagnosed with oligohydramnios if AFI was five or less. ${ }^{3}$ This formed the first group of mothers and the rest formed the second group of mothers. These mothers were included in the final analysis only if then delivered vaginally. Mothers were followed prospectively to assess antenatal risk factors like development of hypertension, abruption placenta, severe anemia. Perinatal outcomes were noted as well.

\section{Data collection and data analysis}

Using a pre-tested semi-structured proforma, demographic information like maternal age and parity were noted. All mothers were asked in detail about their obstetric history. Clinical assessments of the mothers were noted in the proforma. Ultrasound examination was done for all women and four quadrant amniotic fluid volume measurement technique was used to assess the AFI. Perinatal outcomes were noted in the proforma as well. Data was analysed through SPSS version 21. Mean and standard deviation were described for quantitative variables while frequency along with percentages were described for qualitative variables. Difference of proportions were assessed using "N-1" Chi-squared test as recommended by Richardson. ${ }^{4} \mathrm{~A}$ p-value of less than 0.05 was considered statistically significant.

\section{RESULTS}

Of the 200 mothers included in the study, 38 had AFI $\leq 5$. Mean age of mothers with AFI $\leq 5$ was $26.59 \pm 4.76$ years and those with AFI $>5$ was $27.88 \pm 5.12$ years. Most common age group was 20 to 29 years (Table 1 ).

Table 1: Distribution of patients according to their baseline characteristics.

\begin{tabular}{|c|c|c|c|c|c|}
\hline \multirow[t]{2}{*}{ Variables } & \multicolumn{2}{|c|}{ Amniotic fluid index $\leq 5(n=38)$} & \multicolumn{2}{|c|}{ Amniotic fluid index $>5(n=162)$} & \multirow[t]{2}{*}{$P$ value } \\
\hline & $\mathbf{n}$ & $\%$ & $\mathbf{n}$ & $\%$ & \\
\hline \multicolumn{6}{|l|}{ Maternal age (in years) } \\
\hline Less than 20 & 4 & 11 & 32 & 20 & \multirow{3}{*}{$>0.05$} \\
\hline 20 to 29 & 27 & 71 & 86 & 53 & \\
\hline More than 29 & 7 & 18 & 44 & 27 & \\
\hline \multicolumn{6}{|l|}{ Parity } \\
\hline Nulliparous & 22 & 58 & 87 & 54 & \multirow{2}{*}{$>0.05$} \\
\hline Multiparous & 16 & 42 & 75 & 46 & \\
\hline \multicolumn{6}{|l|}{ Gestational age (in weeks) } \\
\hline 28 to 32 & 3 & 8 & 22 & 14 & \multirow{6}{*}{$>0.05$} \\
\hline 32 to 34 & 5 & 13 & 31 & 19 & \\
\hline 34 to 36 & 0 & 0 & 17 & 10 & \\
\hline 36 to 38 & 9 & 24 & 47 & 29 & \\
\hline 38 to 40 & 7 & 18 & 31 & 19 & \\
\hline more than 40 & 14 & 37 & 14 & 9 & \\
\hline \multicolumn{6}{|l|}{ Antenatal risk factors } \\
\hline Pregnancy induced hypertension & 11 & 29 & 19 & 12 & $<0.05$ \\
\hline Intra-uterine growth restriction & 13 & 34 & 17 & 10 & $<0.001$ \\
\hline Abruptio placenta & 5 & 13 & 9 & 6 & 0.13 \\
\hline Severe anemia & 8 & 21 & 14 & 9 & $<0.05$ \\
\hline Prolonged pregnancy & 5 & 13 & 12 & 7 & 0.22 \\
\hline
\end{tabular}


Majority of the mothers were nulliparous (58\% in group with AFI $\leq 5$ and $54 \%$ in group with AFI $>5$ ). Majority of the mothers (37\%) with AFI $\leq 5$ had gestational age more than 40 weeks, while only $9 \%$ of the mothers with AFI > 5 had gestational age greater than 40 weeks. Distribution of mothers according to their age, parity and gestational age was similar in both the groups. Most common antenatal risk factors studies were pregnancy induced hypertension ( $29 \%$ vs $12 \%$; p value <0.05), intrauterine growth restriction (34\% vs $10 \%$; p value $<0.001)$, abruption placenta ( $13 \%$ vs $6 \%$; $\mathrm{p}$ value $=0.13)$, severe anemia ( $21 \%$ vs $9 \%$; p value $<0.05)$ and prolonged pregnancy $(13 \%$ vs $7 \%$; $\mathrm{p}$ value $=0.22)$. Only pregnancy induced hypertension, intra-uterine growth restriction and severe anemia were significantly higher among mothers with AFI less than 5. Perinatal outcomes were studied in both the groups (Table 2).

Table 2: Perinatal outcomes observed in the study.

\begin{tabular}{|c|c|c|c|c|c|}
\hline \multirow[t]{2}{*}{ Variables } & \multicolumn{2}{|c|}{$\begin{array}{l}\text { Amniotic fluid index } \leq 5 \\
(\mathrm{n}=38)\end{array}$} & \multicolumn{2}{|c|}{$\begin{array}{l}\text { Amniotic fluid index }>5 \\
(n=162)\end{array}$} & \multirow[t]{2}{*}{ P value } \\
\hline & $\mathbf{n}$ & $\%$ & $\mathbf{n}$ & $\%$ & \\
\hline Induction of labor & 20 & 53 & 41 & 25 & $<0.05$ \\
\hline Meconium stained liquor & 9 & 24 & 22 & 14 & 0.13 \\
\hline Birth weight less than $2.5 \mathrm{kgs}$ & 21 & 55 & 38 & 23 & $<0.001$ \\
\hline APGAR score less than 7 at 1 minute & 11 & 29 & 31 & 19 & 0.17 \\
\hline APGAR score less than 7 at 5 minutes & 3 & 8 & 7 & 4 & 0.29 \\
\hline NICU admission & 5 & 13 & 12 & 7 & 0.22 \\
\hline
\end{tabular}

Induction of labor was required in $53 \%$ of mothers with oligohydramnios as compared to $25 \%$ in mothers without oligohydramnios, $\mathrm{p}$ value $<0.05$. Though proportion of mothers with meconium stained liquor, birth weight less than $2.5 \mathrm{kgs}$, APGAR score less than 7 and neonatal intensive care unit were higher among mothers with oligohydramnios as compared to those without oligohydramnios, only difference in proportion of births less than $2.5 \mathrm{kgs}$ were significantly higher among mothers with oligohydramnios.

\section{DISCUSSION}

The present single centre study describes the perinatal outcomes associated with oligohydramnios. Reported rates of oligohydramnios are influenced by variations in diagnostic criteria, the population studied, the threshold used, and the gestational age at the time of the ultrasound examination. A study of more than three thousand uncomplicated pregnancies with singleton nonanomalous fetuses between 40 and 41.6 weeks of gestation noted oligohydramnios in $11 \% .^{5}$ The incidence is high in laboring women, largely due to rupture of fetal membranes during or just before labor. ${ }^{6}$ There are both objective and subjective ultrasound criteria for assessing oligohydramnios. Although use of an objective criterion is generally preferable, subjective suspicion of amniotic fluid volume by experienced examiners has similar sensitivity for diagnosing reduced amniotic fluid volume. ${ }^{7}$ In the present study, we used the AFI for diagnosing oligohydramnios. As the 5 th percentile for AFI averages approximately $7 \mathrm{~cm}$ throughout gestation, an AFI less than $5 \mathrm{~cm}$ is greater than two standard deviations below the mean value. ${ }^{8}$ Most studies that found significant morbidity associated with oligohydramnios used an AFI less than $5 \mathrm{~cm}$ as the cutoff. ${ }^{9}$ It is for this reason that using the AFI cut-off of 5 $\mathrm{cm}$ is more clinically relevant for the diagnosis of oligohydramnios, and represents a volume that may require further assessment and/or clinical intervention.

The baseline characteristics of mothers in the present study like age, parity and gestational age were similar in both the groups. We observed significantly higher proportion of mothers with pregnancy induced hypertension, intrauterine growth restriction and severe anemia in mothers with oligohydramnios. Proportion of pregnancies needing induction of labor and birth weight less than $2.5 \mathrm{kgs}$ were higher among mothers with oligohydramnios. In a similar study by Locatelli et al, univariate and multivariate analyses indicated that detecting oligohydramnios in a low-risk pregnancy after 40.0 weeks carried a more than 2 -fold increased risk of a small for gestational age (SGA) neonate. This carries immense clinical importance as they are at significantly increased risk of death in utero. Although the mechanism that leads to amniotic fluid reduction after term is unclear, it has been suggested that low amniotic fluid volume is a manifestation of placental involution, and as such it is a risk factor for fetal growth restriction. ${ }^{10}$ In the present study we did not record the fetal heart rate readings but it is suggested that future studies should assess abnormal fetal heart rate tracings (variable decelerations, late decelerations, or loss of variability) and umbilical cord $\mathrm{pH}$ leading to cesarean delivery.

In the present study we found oligohydramnios not to be associated with presence of meconium-stained amniotic fluid. This has often been debated in the previously published literature. ${ }^{11,12}$ It is to be noted that most of the 
published reports included women with a gestational age beyond 42 weeks, which might have accounted for the discrepancy. Additionally, we did not find any association between APGAR scores and presence of oligohydramnios. This is in contrast to what was reported by Ekin et al who found that patients with oligohydramnios have a significantly higher incidence of low APGAR scores at one and five minutes. ${ }^{13}$ It is plausible that low APGAR scores were commonly encountered in emergency cesarean delivery which were excluded from the study.

There are a few limitations of this study. First is the fact that this is a single centre study and the sample size is small. So, the results of the present study might not be generalizable to others study settings. Second, the clinician taking care of the patient was aware of the AFI, risk factors of pregnancy, and the results of any antenatal testing that was undertaken. This information could have influenced their decision to deliver the patient and the number of intensive care admissions.

\section{CONCLUSION}

In the present study, we included singleton pregnancies with oligohydramnios and compared their perinatal outcomes without oligohydramnios. We observed that induction of labor and low birth weight were significantly associated with oligohydramnios. However, there is insufficient information about the mechanisms behind the pathogenesis of oligohydramnios, for which further studies are required. In addition, prospective randomized trials are needed to establish whether early induction of labor in the presence of a oligohydramnios improves perinatal outcome.

\section{Funding: No funding sources}

Conflict of interest: None declared

Ethical approval: The study was approved by the Institutional Ethics Committee

\section{REFERENCES}

1. Rutherford SE, Phelan JP, Smith CV, Jacobs N. The four-quadrant assessment of amniotic fluid volume: an adjunct to antepartum fetal heart rate testing. Obstet Gynecol. 1987;70(3):353.

2. Rossi AC, Prefumo F. Perinatal outcomes of isolated oligohy- dramnios at term and post-term pregnancy: a systematic review of literature with meta-analysis. Eur J Obstet Gynecol Reprod Biol. 2013;169(2):14954.
3. Phelan JP, Ahn MO, Smith CV, et al. Amniotic fluid index measurements during pregnancy. J Reprod Med. 1987;32(8):601-4.

4. Richardson JT. The analysis of $2 \times 2$ contingency tables-Yet again. Statistics in medicine. 2011;30(8):890.

5. Locatelli A, Zagarella A, Toso L, Assi F, Ghidini A, Biffi A. Serial assessment of amniotic fluid index in uncomplicated term pregnancies: prognostic value of amniotic fluid reduction. J Matern Fetal Neonatal Med. 2004;15(4):233.

6. Sarno AP, Ahn MO, Brar HS, Phelan JP, Platt LD. Intrapartum Doppler velocimetry, amniotic fluid volume, and fetal heart rate as predictors of subsequent fetal distress. I. An initial report. Am J Obstet Gynecol. 1989;161(6):1508.

7. Magann EF, Perry Jr KG, Chauhan SP, Anfanger PJ, Whitworth NS, Morrison JC. The accuracy of ultrasound evaluation of amniotic fluid volume in singleton pregnancies: the effect of operator experience and ultrasound interpretative technique. J Clin Ultrasound. 1997;25(5):249.

8. Moore TR, Cayle JE. The amniotic fluid index in normal human pregnancy. Am J Obstet Gynecol 1990; 162(5): 1168 .

9. Mabie WC, Gonzalez AR, Sibai BM, Amon E. A comparative trial of labetalol and hydralazine in the acute management of severe hypertension complicating pregnancy. Obstet Gynecol 1987; $70(3): 328$.

10. Sylvestre G, Divon MY. The pathophysiology of amniotic fluid volume (AFV) in the postterm pregnancy. American Journal of Obstetrics and Gynecology. 1999 Jan 1;180(1S-II):23S.

11. Rutherford SE, Phelan JP, Smith CV, Jacobs N. The four-quadrant assessment of amniotic fluid volume: an adjunct to antepartum fetal heart rate testing. Obstet Gynecol. 1987;70(3 Pt 1):353-6.

12. Magann EF, Chauhan SP, Kinsella MJ, McNamara MF, Whithworth NS, Morrison JC (1999) Antenatal testing among 1001 patients at high risk: the role of ultrasonographic estimate of amniotic fluid volume. Am J Obstet Gynecol 180(6):1330-6

13. Ekin A, Gezer C, Taner CE, Ozeren M. Perinatal outcomes in pregnancies with oligohydramnios after preterm premature rupture of membranes. J Mat -Fet Neonat Med. 2015;28(16):1918-22.

Cite this article as: Gaware S, Bangal VB. Perinatal outcome in uncomplicated pregnancies. Int J Reprod Contracept Obstet Gynecol 2019;8:215-8. 